\title{
Internal Carotid Artery Occlusion Following Correction of Atypical Aortic Coarctation
}

\author{
-Case Report- \\ Naoyuki ISOBE, Shuichi OKI, Masayuki SUMIDA, Yukari KANOU, \\ Shinya NABIKA, Yosuke WATANABE, and Naomichi UCHIDA* \\ Departments of Neurosurgery and ${ }^{*}$ Cardiovascular Surgery, \\ Hiroshima City Asa Hospital, Hiroshima
}

\begin{abstract}
An 11-year-old boy developed occlusion of the left internal carotid artery (ICA) following surgical correction of atypical coarctation of the aorta. The patient was admitted to our hospital after presenting with severe hypertension secondary to abdominal aortic hypoplasia and renal artery stenosis. Reconstruction of the abdominal aorta with bypass grafting was performed without complication. However, in the postoperative period, the patient experienced recurrent transient ischemic attacks manifesting as paresthesia of the right upper limbs, dysarthria, and right-sided weakness. Cerebral angiography revealed occlusion of the $\mathrm{C}_{2}$ portion of the left ICA and decreased resting cerebral blood flow in the left hemisphere. Extracranial-intracranial arterial bypass was performed, and the patient suffered no further adverse neurological events. Coarctation of the aorta is an uncommon congenital condition that may result in cerebral ischemic disease. The cerebrovascular circulation should be evaluated, even in patients without a pre-existing history of cerebral ischemic symptoms.
\end{abstract}

Key words: cerebrovascular disease, atypical aortic coarctation, extracranial-intracranial bypass

\section{Introduction}

Cerebral ischemia in the pediatric population is usually caused by cardiac thromboembolic disease, infection, trauma, or moyamoya disease. ${ }^{8,11,12,18)}$ Occlusion of the intracranial vessels is less commonly caused by hematologic disorders or coagulopathies, and collagen vascular diseases (such as fibromuscular dysplasia, neurofibromatosis, etc.). ${ }^{7,9,14,16)}$ Arteriosclerosis is an uncommon etiology of cerebral ischemia in children, although a major cause of cerebrovascular disease in adults. Coarctation of the aorta is a congenital malformation that may also generate cerebral ischemia. We treated an 11-year-old boy with occlusion of the left internal carotid artery (ICA) following surgical correction of atypical coarctation of the aorta.

\section{Case Report}

An 11-year-old boy presented to our pediatric hospital on August 25, 2002, with acute onset of

Received May 24, 2004; Accepted March 1, 2005 headache and vomiting. The patient had severe hypertension. He was admitted for further evaluation and treatment. Echocardiography and optic fundus examination detected hypertensive changes, but neither valvular heart disease nor arrhythmia such as atrial fibrillation and sick sinus syndrome. Digital subtraction angiography (DSA) revealed abdominal aortic stenosis, left renal artery stenosis, and a narrowly patent right renal artery (Fig. 1A, B). The diagnosis was renovascular hypertension and atypical coarctation of the aorta. His blood pressure was medically uncontrollable and remained high: systolic blood pressure (SBP) was $160-180 \mathrm{mmHg}$, and diastolic blood pressure (DBP) was 100-110 mmHg.

Surgical reconstruction of the abdominal aorta and both renal arteries was performed. During the operation, blood pressure was controlled at SBP/DBP 90-120/50-80 mmHg, and no paroxysmal atrial fibrillation occurred. Blood pressure was controlled within normal limits, but hypotensive medication was subsequently given. Although the patient experienced postoperative transient rightsided weakness, his condition had improved. DSA 

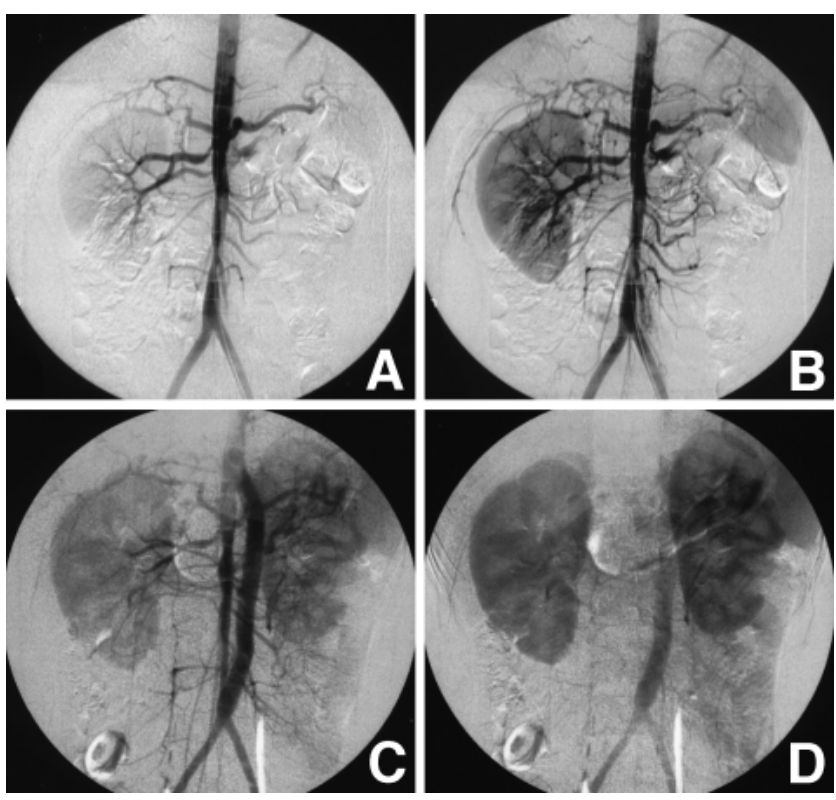

Fig. 1 Abdominal angiograms showing abdominal aortic stenosis, left renal artery stenosis, and a narrowly patent right renal artery $(A$, $B$ ), with correction of renal perfusion (seen here as enhancement of the renal parenchyma) following surgical vascular reconstruction (C, D).

demonstrated adequate graft patency and improvement in renal blood flow (Fig. 1C, D). A regimen of nifedipine, valsartan, and aspirin was initiated, and he was discharged from the hospital.

The patient returned on November 3, 2002 after developing recurrent transient ischemic attacks (TIAs), manifesting as several minutes duration of dysarthria, right upper extremity numbness, and right-sided weakness. Computed tomography (CT) revealed low density areas in the left caudate nucleus and periventricular region (Fig. 2), and the patient was re-hospitalized. The right-sided weakness resolved within 1 day. Blood pressure and lipid profile remained within normal limits, and serum markers of inflammation were generally unremarkable. Fluid-attenuated inversion recovery magnetic resonance (MR) images obtained at the first hospitalization (i.e. September 2, 2002) had shown a small ischemic area in the left periventricular region and MR angiography showed decreased blood flow in the $\mathrm{C}_{1}$ and $\mathrm{C}_{2}$ portions of the left ICA. However, these findings were not then considered significant, because the peripheral vessels were well visualized.

Medical treatment with intravenous ozagrel hydrochloride and low molecular dextran was instituted. Cerebral angiography on November 6, 2002 revealed obstruction of the left ICA distal

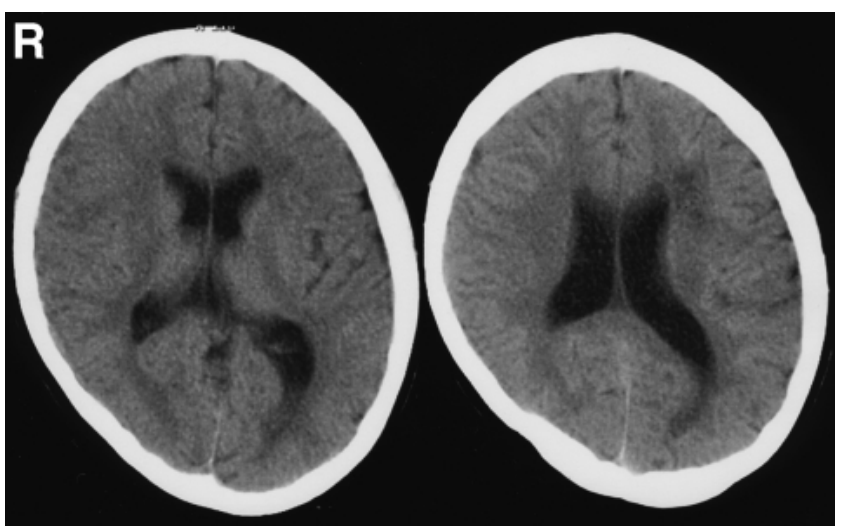

Fig. 2 Computed tomography scans obtained on re-hospitalization demonstrating low density areas in the left caudate nucleus and periventricular region.

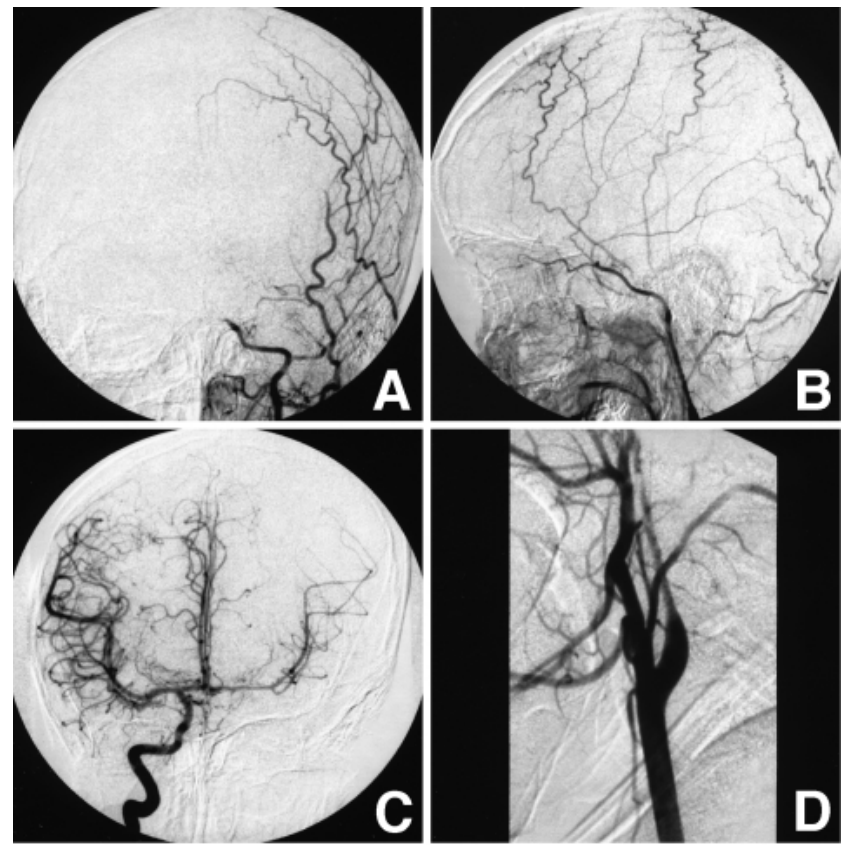

Fig. 3 Left common carotid angiograms showing occlusion of $C_{1}$ portion of the internal carotid artery (ICA) (A, B), and right common carotid angiogram demonstrating blood supply to the left hemisphere via the anterior communicating artery (C). Left common carotid angiogram showing narrowing of the cervical portion of the ICA (D).

to the ophthalmic artery and collateral filling via the contralateral anterior communicating artery (Fig. 3A-C), but no aortic stenosis. The cervical portion of the left ICA was narrowed, beginning 

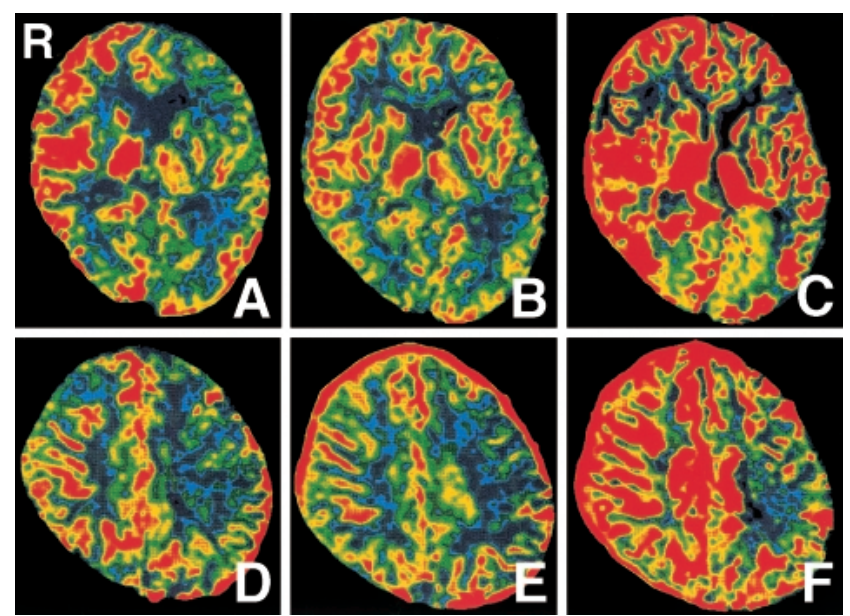

Fig. 4 Xenon computed tomography (CT) scans before surgery indicating decreased cerebral blood flow (CBF) in the left hemisphere (A, $D)$, and xenon CT scans after surgery showing increased CBF in the left hemisphere, and normalization of the cerebrovascular reserve capacity $(\mathrm{B}, \mathrm{C}, \mathrm{E}, \mathrm{F})$.

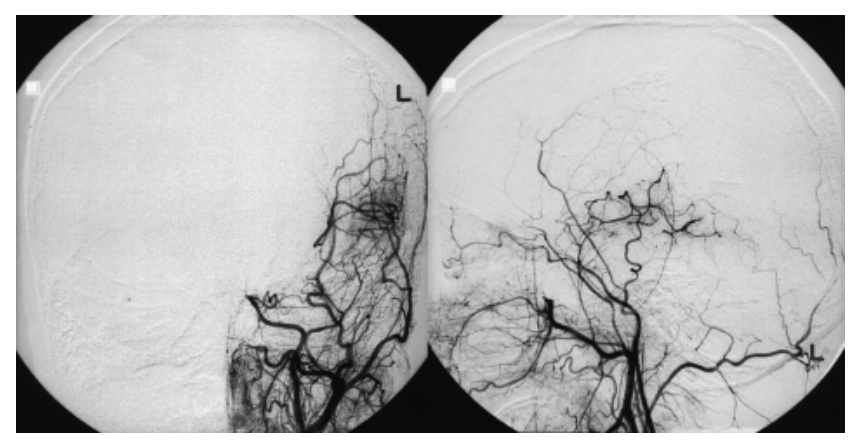

Fig. 5 Left common carotid angiograms after surgery showing adequate perfusion in the territory supplied by the middle cerebral artery.

slightly distal to the carotid bifurcation (Fig. 3D). Xenon CT showed decreased cerebral blood flow (CBF) in the left hemisphere. The resting regional $\mathrm{CBF}$ (rCBF) was $37.3-53.1 \mathrm{ml} / \mathrm{min} / 100 \mathrm{~g}$ in the right hemisphere, and $24.8-33.2 \mathrm{ml} / \mathrm{min} / 100 \mathrm{~g}$ in the left (Fig. 4A, D). Acetazolamide loading $(10 \mathrm{mg} / \mathrm{kg})$ was also performed, but the patient began vomiting in the middle of the examination. Therefore, the regional cerebrovascular reactivity (rCVR) could not be calculated.

Left superficial temporal artery to middle cerebral artery anastomosis was performed to treat the cerebral ischemia (Fig. 5). The patient tolerated the procedure well and was discharged from the hospital 1 month later without postoperative neurological deficits. Xenon CT revealed increased CBF in the left hemisphere and normalization of the cerebrovascular reserve capacity. The resting $\mathrm{rCBF}$ was $25.2-39.5 \mathrm{ml} / \mathrm{min} / 100 \mathrm{~g}$ in the left hemisphere, and the rCVR was $32.4-80.2 \%$ (Fig. 4B, C, E, F). The patient remained free of neurological events at follow up 10-month after the surgery.

\section{Discussion}

Coarctation of the aorta is an important congenital malformation that can cause obstruction in various vascular beds. Typical coarctation causes strictures in the isthmus of the thoracic aorta and accounts for the overwhelming majority (98\%) of this type of malformation. Atypical coarctation is rare and may be subisthmic, that is located in the region from the descending to the abdominal aorta, or preisthmic, that is located in the region between the ascending aorta and the aortic arch. ${ }^{3,4,6,10)}$ Coarctation of the aorta often extends to other vascular structures, such as stenosis of the left common carotid artery, left subclavian artery, or intracranial arteries. Atypical coarctation of the aorta with cerebral arterial occlusion is not so common..$^{1,15)}$

The etiology of atypical coarctation of the aorta remains unknown, although association with neurofibromatosis, fibromuscular dysplasia, and Turner syndrome have been reported.2,13,17) No such etiology was found in our patient. Inflammatory states, such as aortitis, have been also implicated in the development of atypical coarctation of the aorta. However, histological examination of the excised left renal artery in this case revealed hypoplasia of the vessel without degeneration or inflammation, suggesting that the present case was a true congenital malformation.

The main causes of cerebral arterial occlusion are arteriosclerosis, embolism, vasculitis, etc. In this case, arteriosclerosis was excluded, because the patient was 11 years old and histological study of the renal artery demonstrated hypoplasia of the vessel without arteriosclerotic change. The patient had no past history of cardioembolism, such as atrial fibrillation or valvular heart disease. Embolus was formed during the first surgery performed on the abdominal aorta, but embolism generally occurs in the arteries of the leg rather than the carotid artery.

MR angiography before reconstruction of the abdominal aorta showed stenosis of the left ICA $\left(\mathrm{C}_{1}\right.$ and $\mathrm{C}_{2}$ portions). The SBP/DBP was high between 160-180/100-110 mmHg, even after administration of two or more hypotensive agents. The SBP/DBP was reduced to $90-120 / 50-80 \mathrm{mmHg}$ during the 
operation and was subsequently controlled within normal limits. Therefore, the postoperative appearance of clinical symptoms of ICA obstruction probably occurred as the result of reduced CBF secondary to normalization of blood pressure following the first procedure.

Cerebral angiography performed after repeated TIAs showed that the cervical portion of the left ICA was narrowed, beginning slightly distal to the carotid bifurcation and stenosed after the branching of the ophthalmic artery. Repeat cerebral angiography 2 months later showed the same findings. In contrast, narrowing of the left ICA in the acute phase of embolism would be expected to progress further. Therefore, the narrowing of the cervical ICA had been present before the first surgery.

Conservative treatment is often the best choice in the context of pediatric cerebrovascular disease, as the potential for forming collateral circulatory routes and recanalization of the original obstruction is high.5) Our patient was 11 years old, so the development of collateral circulation from the ophthalmic artery and external carotid artery could be expected. However, the patient suffered frequent TIAs for a few days, the resting rCBF was decreased in the left hemisphere, and electroencephalography (EEG) with hyperventilation showed slow waves in the left hemisphere. Furthermore, stenosis could be expected in the contralateral ICA, with the risk of hemodynamic ischemic stress. Consequently, extracranial-intracranial bypass was performed to improve the intracranial hemodynamics. The patient did not experience further neurological events.

The present case emphasizes the importance of evaluating cerebrovascular perfusion in patients with atypical coarctation of the aorta, even if no pre-existing neurological symptoms are present. Postoperative symptoms consistent with intracranial stenosis or obstruction require immediate evaluation (e.g., CBF study, EEG, etc.), and surgical reperfusion procedures should be considered.

\section{References}

1) Baltaxe HA, Bloch S, Mooring PK: Coarctation of the thoracic aorta associated with cerebral arterial occlusive disease. AJR Am J Roentgenol 139: 1226-1229, 1982

2) Bloor K, Williams RT: Neurofibromatosis and coarctation of the abdominal aorta with renal artery involvement. Br J Surg 50: 811-813, 1963

3) Cohen JR, Birnbaum E: Coarctation of the abdominal aorta. J Vasc Surg 8: 160-164, 1988

4) DeBakey ME, Garrett HE, Howell JF, Morris GC Jr: Coarctation of the abdominal aorta with renal arterial stenosis: surgical considerations. Ann Surg 165: 830-843, 1967

5) Fuwa I, Matsukado Y, Kodama T, Yokota A, Kajiwara H: [Cerebrovascular occlusive disease in children - a clinical study with non-moyamoya disease]. No To Hattatsu 18: 134-139, 1986 (Jpn, with Eng abstract)

6) Hallett JW Jr, Brewster DC, Darling RC, O'Hara PJ: Coarctation of the abdominal aorta: current options in surgical management. Ann Surg 191: 430-437, 1980

7) Lemahieu SF, Marchau MMB: Intracranial fibromuscular dysplasia and stroke in children. Neuroradiology 18: 99-102, 1979

8) Lemesle M, Manceau E, Osseby GV, MadinierChappart N, Moreau T, Giroud M: [Ischemic cerebrovascular stroke of arterial origin in the child]. Rev Neurol (Paris) 157: 1255-1263, 2001 (Fre, with Eng abstract)

9) Levisohn PM, Mikhael MA, Rothman SM: Cerebrovascular changes in neurofibromatosis. Dev Med Child Neurol 20: 789-792, 1978

10) Paroni R, Astuni M, Baroni C, Giavazzi T, Luppi C, Pisa G, Tognoli S, Zola C: Abdominal aortic coarctation inducing aortic occlusion and renovascular hypertension. J Cardiovasc Surg 32: 770-774, 1991

11) Raybaud CA, Livert $M$, Jiddane $M$, Pinsard $N$ : Radiology of ischemic strokes in children. Neuroradiology 27: 567-578, 1985

12) Satoh S, Shirane R, Yoshimoto T: Clinical survey of ischemic cerebrovascular disease in children in a district of Japan. Stroke 22: 586-589, 1991

13) Simpson JW, Nora JJ, Singer DB, McNamara DG: Multiple valvular sclerosis in Turner phenotypes and Rubella syndrome. Am J Cardiol 23: 94-97, 1969

14) Slagsvold JE, Bergsholm P, Larsen JL: Fibromuscular dysplasia of intracranial arteries in a patient with multiple enchondromas (Ollier disease). Neurology 27: 1168-1171, 1977

15) Smith SH, Kelly DR: Coarctation of the abdominal aorta in a child: morphometric analysis of the arterial lesion. Pediatr Pathol 5: 363-371, 1986

16) Tomsick TA, Lukin RR, Chambers AA, Benton C: Neurofibromatosis and intracranial arterial occlusive disease. Neuroradiology 11: 229-234, 1976

17) Vuong PN, Janzen J, Bical O, Susa-Uva M: Fibromuscular dysplasia causing atypical coarctation of the thoracic aorta: histological presentation of a case. Vasa 24: 194-198, 1995

18) Wanifuchi $H$, Kagawa $M$, Takeshita $M$, Izawa $M$, Kitamura K: Ischemic stroke in infancy, childhood, and adolescence. Childs Nerv Syst 4: 361-364, 1988

Address reprint requests to: N. Isobe, M.D., Department of Neurosurgery, Hiroshima City Asa Hospital, 2-1-1 Kabeminami, Asakita-ku, Hiroshima 731-0293, Japan. 